\title{
NONLINEAR MAGNETIC PROPERTIES OF MAGNETIC FLUIDS FOR AUTOMOTIVE APPLICATIONS
}

\author{
BARNABÁS HORVÁTH*1 AND ISTVÁN SZALAI ${ }^{2}$ \\ ${ }^{1}$ Institute of Physics and Mechatronics, University of Pannonia, Egyetem utca 10, Veszprém H-8200, \\ HUNGARY \\ ${ }^{2}$ Institute of Mechatronics Engineering and Research, University of Pannonia, Gasparich Márk utca 18/A, \\ Zalaegerszeg H-8900, HUNGARY
}

\begin{abstract}
The external magnetic field required to activate a magnetic fluid in an industrial application is sufficiently large that magnetization is no longer a linear function of the external field strength, i.e. magnetic fluids exhibit nonlinear characteristics. The aim of our research was to develop a measuring system which is capable of determining the nonlinear AC susceptibility of magnetic fluids at discrete frequencies and in the presence of a high-intensity driving magnetic field. The measurement of susceptibility is based on the determination of the change in frequency of a low-intensity field, which is generated by an LC oscillator. The application of sinusoidal excitation to the material results in a variation in the susceptibility that modulates the frequency of the measured low-intensity field and in the appearance of higher-order harmonics of the driving field. The higher-order components of the nonlinear AC susceptibility are extracted from the measured response by Fourier analysis. By applying the measuring system, the nonlinear susceptibility of water-based ferrofluids (Ferrotec's EMG 700) and its dependence on the magnetic field strength were investigated.
\end{abstract}

Keywords: AC susceptometry, nonlinear susceptibility, ferrofluid

\section{Introduction}

Regarding automotive applications, one of the most important characteristics of magnetic fluids is their behavior in magnetic fields. The majority of these applications are for seals [1], vibration damping [2] and torque transmission [3]. In most cases, the magnitude of an external magnetic field required to activate a fluid is on the scale where the $\mathbf{M}$ magnetization is no longer a linear function of the $\mathbf{H}$ field strength. This means that magnetic fluids show nonlinear characteristics, and the $\chi=\partial M / \partial H$ susceptibility depends on the magnetic field strength. In the case of magnetic fluids, the nonlinearity is the result of two effects: normal saturation (alignment of the magnetic dipole moments) and formation of structures (particle chains). The latter influences the susceptibility as the magnetic field shifts the equilibrium between the structures with different dipole moments (single particles and particle chains of different sizes).

In a weak time-varying $H_{\mathrm{e}}(t)=H_{\mathrm{e} 0} \sin (\omega t)$ magnetic field (where $t$ denotes the time and $\omega=2 \pi f$ represents the angular frequency), the magnetization also changes periodically but lags behind the magnetic field because reorientation of the magnetic dipole moments is not instantaneous. In this case, the dynamic magnetic susceptibility can be defined as a complex quantity $\chi^{*}(\omega)=$

\footnotetext{
*Correspondence: bhorvath@almos.uni-pannon.hu
}

$\chi^{\prime}(\omega)-i \chi^{\prime \prime}(\omega)$. The real part, $\chi^{\prime}$, is related to the reversible magnetization process and it is in-phase with the alternating field. Within the low-frequency limit, $(f \rightarrow 0)$ $\chi^{\prime}$ approaches the initial gradient of the steady-state magnetization (initial DC susceptibility). If $f \rightarrow \infty$, the reorientation of the magnetic dipole moments cannot follow the alternating field and $\chi^{\prime}$ approaches zero. The imaginary, out-of-phase component, $\chi^{\prime \prime}$, is proportional to power losses due to energy absorption from the field and peaks at a characteristic frequency, $f_{\mathrm{c}}$.

However, if the amplitude of the alternating field is sufficiently large, higher-order harmonics appear in the magnetization due to the nonlinear characteristics. In this case, the real part of the alternating current (AC) magnetic susceptibility is:

$$
\chi^{\prime}=\chi_{0}+\chi_{2 \omega} \cos (2 \omega t)+\chi_{4 \omega} \cos (4 \omega t)+\ldots,
$$

where $\chi_{0}$ denotes the base component, and $\chi_{2 \omega}$ and $\chi_{4 \omega}$ represent the amplitude of the second- and fourth-order harmonics, respectively [4]. A similar equation holds for the imaginary component, $\chi^{\prime \prime}$. In this work, only the harmonics of the real part are considered, because the nonlinear susceptibility at frequencies much higher than the characteristic frequency of the magnetic fluid is investigated, where the imaginary part is very close to zero. By taking magnetic fluids into consideration, the amplitudes of sixth- and higher-order harmonics are so small 


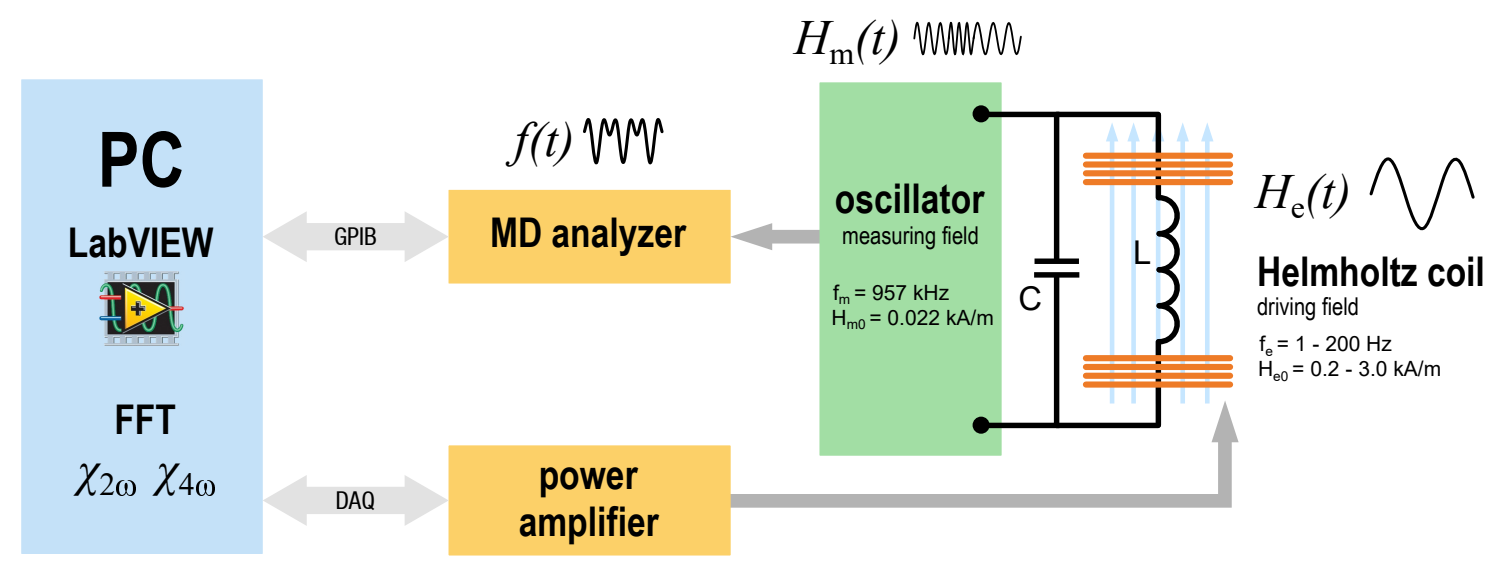

Figure 1: A block diagram of the nonlinear magnetic measuring system.

that they can be neglected. In alternating magnetic fields, only the even-order harmonics appear, because the magnetization of magnetic fluids exhibits inversion symmetry with respect to a change in the direction of $\mathbf{H}$. Should a symmetry-breaking DC bias field be superimposed on the AC magnetic field, both the odd- and even-order harmonics appear. As the nonlinearity is caused partly by structure formation, the components of the nonlinear susceptibility and their ratios are sensitive to changes in the structure of magnetic fluids.

Numerous studies have discussed the theoretical description of the nonlinear susceptibility of magnetic fluids [4-7], but experimental results are rather scarce. Nevertheless, nonlinear magnetic susceptibility measurements are quite useful for the study of magnetic fluids and provide additional information besides linear AC susceptometry. If the linear response to small-amplitude fields is measured, the relaxation processes of the magnetic dipoles can be studied. However, by applying the nonlinear method, it is possible to obtain information about the structural evolution of an activated magnetic fluid.

The aim of our work was to develop a measuring system which is capable of determining the nonlinear AC susceptibility of magnetic fluids at discrete frequencies in the presence of external magnetic fields. The dependence of the 2nd- and 4th-order nonlinear AC susceptibility of ferrofluids on the magnetic field strength was investigated by the developed susceptometer.

\section{Experimental}

\subsection{Nonlinear measurement method and setup}

The measurement method of susceptibility is based on our nonlinear dielectric system [8], which is adapted to magnetic measurements. With this method, the real part of complex AC susceptibility is determined from the change in frequency of a low-intensity measuring field $\left(H_{\mathrm{m}}\right)$. An air core solenoid $(L)$ was filled with the sample of fluid, and the inductance determined the frequency of the sinusoidal measuring field generated by an LC oscillator (Fig. 1). Therefore, any change in the susceptibility of the sample caused the resonance frequency $f_{\mathrm{m}}$ to shift. The susceptibility of the sample was measured at this base frequency. Inside the solenoid, the amplitude of the measuring field is so small $\left(H_{\mathrm{m} 0}=0.022 \mathrm{kA} / \mathrm{m}\right)$ that within this region, the magnetization curve of the fluid can be regarded as linear, thus no significant structural change can be expected.

The oscillator which generated the measuring field was a Colpitts-type parallel LC circuit. The active feedback element was a double triode vacuum tube (ECC88). The capacitor bank $C$ was composed of high-quality silver mica capacitors with a low temperature coefficient. The measuring coil $L$ was connected in parallel with the capacitor block, and by changing the coil, the base frequency of the oscillator could be varied. With different inductors, the measuring frequency was set to discrete values, namely $153 \mathrm{kHz}, 590 \mathrm{kHz}$ and $957 \mathrm{kHz}$. The dimensions of the measuring coils were identical: $25 \mathrm{~mm}$ in length with an inner diameter of $7.1 \mathrm{~mm}$. They were composed of enameled copper wires of different sizes and wound on coil formers made of plexiglass. The resonance frequency of the LC oscillator was measured by a Hewlett Packard 53310A Modulation Domain (MD) Analyzer. The relationship between the resonance frequency and susceptibility of the sample was determined by calibration using different materials of known susceptibility.

A high-intensity driving field $\left(H_{\mathrm{e}}\right)$ was generated by a pair of Helmholtz coils which were placed around the measuring coil. The axis of the Helmholtz coil and, therefore, the direction of the field, were parallel to the axis of the solenoid. The upper limit on the amplitude of the driving magnetic field with the current setup was $H_{\mathrm{e} 0}=6.4 \mathrm{kA} / \mathrm{m}$, which was two orders of magnitude greater than the amplitude of the measuring magnetic field. The maximum of $H_{\mathrm{e} 0}$ depended on the frequency of the field: as the frequency increased, the maximum of $H_{\mathrm{e} 0}$ decreased. The uniformity of the magnetic field strength in the volume of the sample was better than $1 \%$. The Helmholtz coil was driven by a high-current function generator, which consisted of a Labworks PA-138 linear 
power amplifier and a signal source. The input signal of the amplifier was provided by a multifunction data acquisition (DAQ) card (National Instruments PCI-6052E). Any arbitrary waveform could be generated by the signal generator, and the waveform offset by a DC value.

Under the influence of the high-intensity driving field, the susceptibility of the sample changes, therefore, the frequency of the measuring field was modulated. If the driving field is sinusoidal, then the time-domain susceptibility response will contain the higher-order harmonics of the field. The 2nd- and 4th-order components of the nonlinear AC susceptibility were extracted from the measured response by Fourier analysis. The Fast Fourier Transform (FFT) algorithm was implemented using custom-developed LabVIEW software, which provided control and data acquisition functions of the measuring system.

\subsection{Supplemental measurements}

During the nonlinear measurements, the change in susceptibility relative to the zero-field susceptibility was measured, so the real part of the AC susceptibility of the fluid at the base frequency had to be determined by another method. For this purpose, a spectrum was obtained within the frequency range of $200 \mathrm{~Hz}-1 \mathrm{MHz}$. The initial DC susceptibility of the ferrofluid was determined from the DC magnetization curve. The AC susceptibility in zero field was measured by an inductive method where the inductance of a solenoid filled with the sample was measured by an impedance analyzer (Agilent 4284A). By determining the impedances of the empty air core solenoid and when it is filled with the sample, $\chi^{\prime}$ was calculated. The solenoid used for these measurements was the same as the measuring coil of the nonlinear setup.

\subsection{Material}

By applying the nonlinear magnetic measuring system, the nonlinear properties of Ferrotec's EMG 700 ferrofluid were investigated. This material is water-based and contains magnetite particles with a nominal diameter of $\sim 10$ $\mathrm{nm}$. The volume concentration of the particles is 5.8 $\%(\mathrm{v} / \mathrm{v})$. The fluid is stabilized by an anionic surfactant. For the measurements, glass-tube sample holders with an inner diameter of $3.1 \mathrm{~mm}$ were filled with the ferrofluid. The length of the tube extended beyond the length of the measuring coil at both ends. The volume of the sample was $0.49 \mathrm{~cm}^{3}$.

\section{Results and Discussion}

According to the DC magnetization curve, the initial DC susceptibility of the EMG 700 ferrofluid was $\chi_{\mathrm{DC}}=$ 12.57. This is the limiting value of the real part of the complex susceptibility if $f$ approaches zero. The AC susceptibility spectrum of the ferrofluid is shown in Fig. 2. Relaxation of $\chi^{\prime}$ was observed within the lower frequency

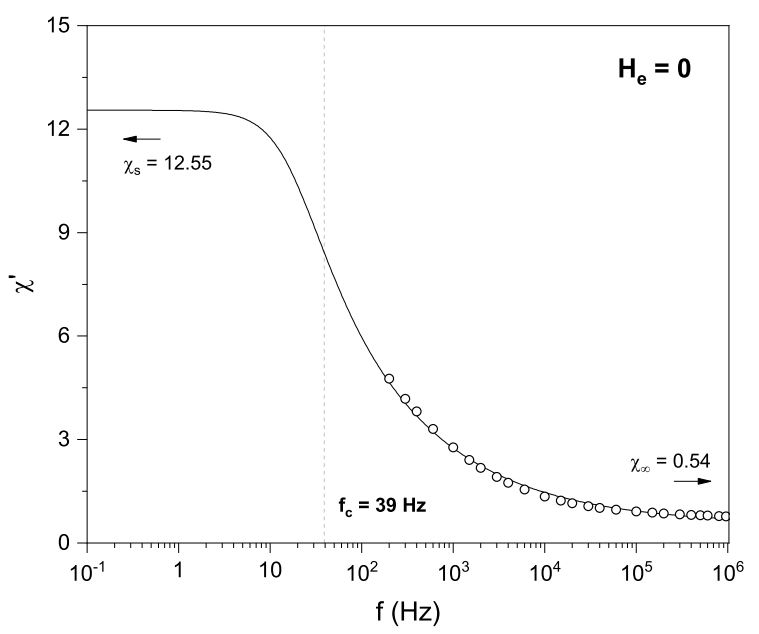

Figure 2: Relaxation of the real part of AC susceptibility in the absence of a driving magnetic field (hollow symbols) by applying the Cole-Cole equation (solid line) together with the estimated parameters of the relaxation.

region. The experimental frequency-dependent susceptibility data was fitted by the Cole-Cole equation (solid line in Fig. 2). This relaxation model is suitable for describing the response of dipoles to an alternating field should the relaxation not be ideal (e.g. if instead of a single relaxation time, a distribution of relaxation times exists).

The fitting process yielded the static $(f \rightarrow 0) \chi_{\mathrm{s}}=$ $12.55 \pm 0.07$ and infinite frequency $(f \rightarrow \infty) \chi_{\infty}=$ $0.54 \pm 0.02$, susceptibilities and the so-called central characteristic time of the relaxation $\left(\tau_{0}\right)$. The reciprocal of the characteristic time yielded the characteristic frequency $f_{\mathrm{c}}=1 /\left(2 \pi \tau_{0}\right)$, which is $39 \pm 5 \mathrm{~Hz}$ for the ferrofluid EMG 700. The spectrum shows that relaxation occurs at much lower frequencies than the base frequency of the nonlinear susceptibility measurements $\left(f_{\mathrm{c}}=39 \mathrm{~Hz}\right.$ vs. $\left.f_{\mathrm{m}}=957 \mathrm{kHz}\right)$. At the high-frequency end $(\sim 1 \mathrm{MHz})$ of the spectrum, $\chi^{\prime}$ decreased to $\sim 0.8$ and changed slightly as the frequency increased in this region. At such a large distance from $f_{\mathrm{c}}$, the imaginary part of the complex susceptibility was close to zero, thus it is justified to consider only the real part (see Eq. 1).

To investigate the nonlinear AC susceptibility, sinusoidal excitation was applied. The frequency of the driving magnetic field was changed from $1 \mathrm{~Hz}$ to $200 \mathrm{~Hz}$ with an amplitude as high as $H_{\mathrm{e} 0}=3 \mathrm{kA} / \mathrm{m}$ (depending on $f_{\mathrm{e}}$ ). The nonlinear AC susceptibility was measured at $957 \mathrm{kHz}$ in all cases. At $H_{\mathrm{e} 0}=0$, the real part of the AC susceptibility of the ferrofluid at the measurement frequency of $f_{\mathrm{m}}=957 \mathrm{kHz}$ was $\chi^{\prime}=0.78$. Fig. 3 shows a typical susceptibility response of the ferrofluid EMG 700 at an excitation frequency of $f_{\mathrm{e}}=1 \mathrm{~Hz}$ and using different magnetic field strengths. It can be seen that the response contained higher-order harmonics. The magnetic field always caused a decrease in the susceptibility (because of the saturation) regardless of its direction. This inversion symmetry was expressed by the fact that the susceptibility response during the first half period 


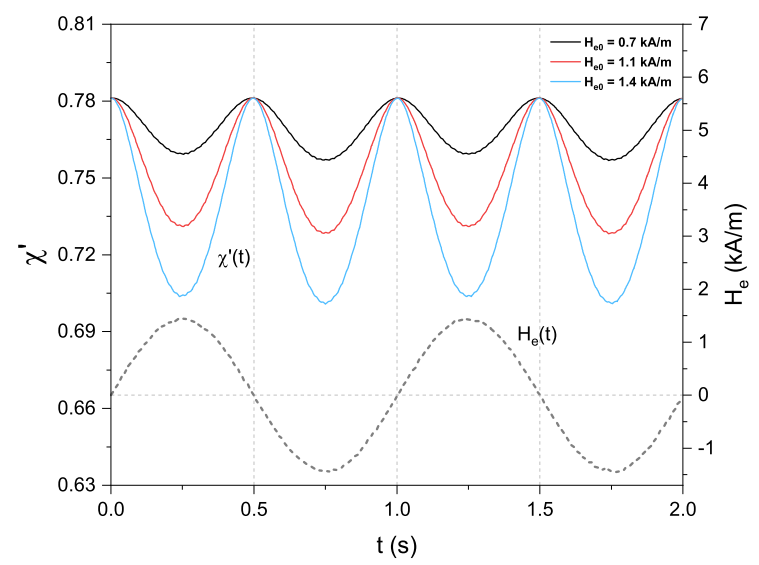

Figure 3: The susceptibility response of the ferrofluid EMG 700 at various driving field amplitudes $\left(f_{\mathrm{e}}=1 \mathrm{~Hz}\right)$.

of $H_{\mathrm{e}}(t)$ was the same as during the second half even if the direction of the field was reversed. Therefore, the maxima of the susceptibility responses were always 0.78 , which corresponded to the point when $H_{\mathrm{e}}(t)$ intersected the value zero.

The dependence of the 2nd- and 4th-order harmonics ( $\chi_{2 \omega}$ and $\chi_{4 \omega}$ in Eq. 1) on the magnetic field strength extracted from the measured response is shown in Figs. 4a and $4 \mathrm{~b}$. At a given driving field strength, $\chi_{2} \omega$ was one order of magnitude larger than $\chi_{4 \omega}$. The amplitude of both components increased as the magnetic field strength rose (at a given driving field frequency). This occurred be-

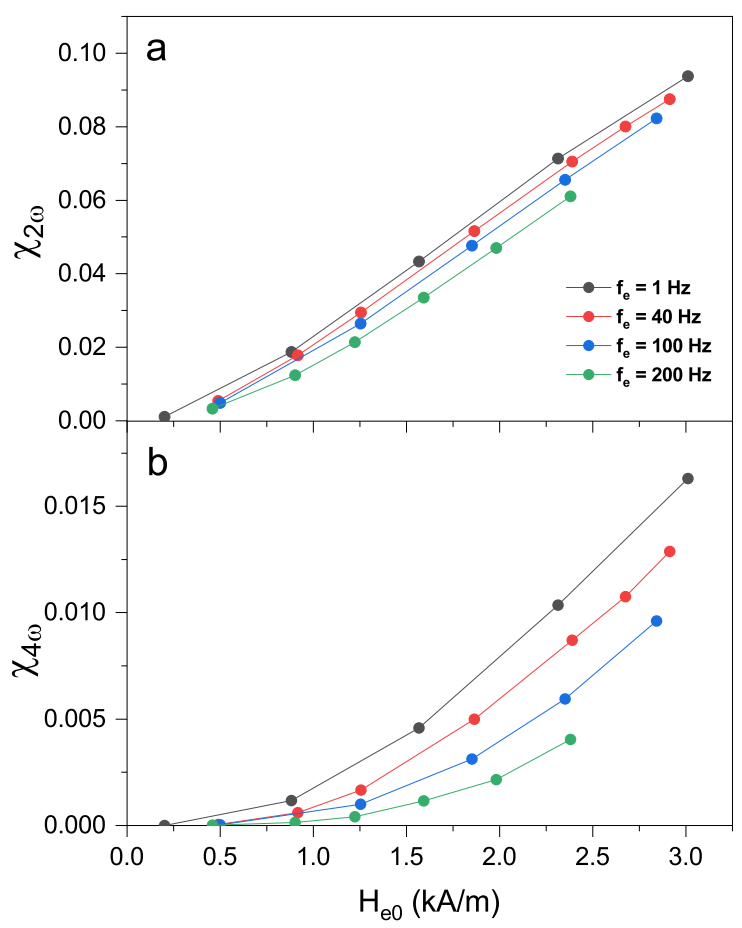

Figure 4: The dependence of the components $\chi_{2 \omega}$ (a) and $\chi_{4 \omega}$ (b) of the nonlinear susceptibility of the ferrofluid EMG 700 on the amplitude of the driving field at different driving field frequencies. cause at higher magnetic field strengths, the nonlinearity of the magnetization curve increased as the magnetization approached the saturation level.

\section{Conclusions}

A nonlinear magnetic measuring system was developed to determine the nonlinear AC susceptibility of magnetic fluids. The system was applied to measure the components of the nonlinear susceptibility of the water-based ferrofluid EMG 700 up to the 4th order. It has been shown that by using the aforementioned system, it is possible to determine the dependence of the nonlinear susceptibility on the magnetic field strength. If the excitation frequency is varied, the relaxation of the nonlinear susceptibility can be also studied. Our future aim is to investigate this relaxation and other types of ferrofluids (based on organic carrier and polydisperse fluids), moreover, to study the influence of the magnetite concentration on the nonlinear magnetic properties.

\section{Acknowledgments}

This project was supported by the European Union and co-financed by the European Social Fund. EFOP-3.6.216-2017-00002.

\section{REFERENCES}

[1] Ravaud, R.; Lemarquand, G.: Mechanical Properties of a Ferrofluid Seal: Three-Dimensional Analytical Study based on the Coulombian Model, Prog. Electromagn. Res. B, 2009, 13, 385-407, DOI: 10.2528/PIERB09020601

[2] Sassi, S.; Cherif, K.; Mezghani, L.; Thomas, M.; Kotrane, A.: An innovative magnetorheological damper for automotive suspension: from design to experimental characterization, Smart Mater. Struct., 2005, 14(4), 811-822, DOI: 10.1088/0964$1726 / 14 / 4 / 041$

[3] Rizzo, R.: An innovative multi-gap clutch based on magneto-rheological fluids and electrodynamic effects: magnetic design and experimental characterization, Smart Mater. Struct., 2017, 26(1), 015007, DOI: 10.1088/0964-1726/26/1/015007

[4] Wang, G.; Huang, J.P.: Nonlinear magnetic susceptibility of ferrofluids, Chem. Phys. Lett., 2006, 421(4), 544-548, DOI: 10.1016/j.cplett.2006.02.010

[5] Huke, B.; Lücke, M.: Magnetic properties of colloidal suspensions of interacting magnetic particles, Rep. Prog. Phys., 2004, 67(10), 1731-1768, DOI: 10.1088/0034-4885/67/10/R01

[6] Szalai, I.; Nagy, S.; Dietrich, S.: Linear and nonlinear magnetic properties of ferrofluids, Phys. Rev. E, 2015, 92(4), 042314, DOI: 10.1103/PhysRevE.92.042314 
[7] Ivanov, A.O.; Kuznetsova, O.B.: Magnetic properties of dense ferrofluids, J. Magn. Magn. Mater., 2002, 252, 135-137, DOI: 10.1016/S03048853(02)00639-X

[8] Horváth, B.; Szalai, I.: Structure of electrorhe- ological fluids: A dielectric study of chain formation, Phys. Rev. E, 2012, 86(6), 061403, DOI: 10.1103/PhysRevE.86.061403 